\title{
Mortalidade neonatal em Taubaté: um estudo caso-controle
}

\author{
Neonatal mortality in Taubaté, São Paulo, Brazil: a case-control study
}

Ruth Sampaio Paulucci', Luiz Fernando C. Nascimento

\section{RESUMO}

Objetivo: Identificar e quantificar os fatores de risco para óbito neonatal em Taubaté, São Paulo.

Métodos: Trata-se de estudo caso-controle com dados de nascidos vivos e de óbitos neonatais de Taubaté, em 2003, obtidos da Secretaria Estadual da Saúde de São Paulo. Os casos (óbitos neonatais) e os controles (recém-nascidos nos mesmos dias daqueles que faleceram) foram reunidos num banco por meio da técnica de linkage. As variáveis independentes foram: variáveis sociodemográficas e assistenciais (idade e escolaridade maternas, paridade, consultas no pré-natal, tipo de parto e relato de natimorto) e variáveis biológicas (peso ao nascer, idade gestacional, escore de Apgar, presença de defeito congênito e sexo). Utilizou-se a regressão logística para identificar e quantificar os efeitos destas variáveis em relação ao óbito neonatal pelo programa SPSS 10.0. Foram introduzidas no modelo as variáveis que apresentaram $p<0,20$ na análise univariada $\mathrm{e}$ permaneceram aquelas $\operatorname{com} p<0,05$.

Resultados: Foram analisados 392 recém-nascidos, sendo 34 óbitos. Havia 198 do sexo masculino (51\%), 60 com baixo peso (15\%) e 51 prematuros (13\%). A idade materna média foi 26 anos. As variáveis "baixo peso ao nascer", "Apgar $<8$ " e "presença de defeito congênito" associaram-se significativamente à presença de óbito neonatal. $\mathrm{O}$ modelo explicou 58\% dos óbitos, mostrando acurácia de $93 \%$.

Conclusões: Os fatores de risco encontrados mostram a importância da prevenção do baixo peso e do bom atendimento na sala de parto, tendo o modelo mostrado boa acurácia e bom poder explicativo para o óbito neonatal.

Palavras-chave: mortalidade neonatal; defeitos congênitos; recém-nascido de baixo peso; fatores de risco.

\section{ABSTRACT}

Objective: To identify and to estimate the risk factors associated to neonatal mortality in Taubaté, São Paulo, Brazil.

Methods: This case-control study enrolled live births in the city of Taubate during 2003. Live birth data and death records were obtained from São Paulo Health Department. Neonatal deaths were cases and babies born alive in the same day of cases were the controls. A single data file was created by linkage approach. Dependent variable was neonatal death. Independent variables were those related to socio-demographic characteristics and prenatal care (maternal age, years in school, parity, previous stillbirths, prenatal care), as well as the biological ones (birthweight, gender, gestational age, congenital defects and Apgar score). Logistic regression was used to identify and to estimate the risk factors associated to neonatal death. The variables with $p<0.20$ were introduced in the model and maintained if $p<0.05$, by SPSS 10.0.

Results: 392newborns with 34 neonatal deaths were studied. There were 198 males (50\%), 60 low birth weight $(15 \%)$ and 51 preterm infants (13\%). Mean maternal age was 26 years. The variables "low birth weight", "Apgar score $<8$ " and "congenital defects" were significantly associated to neonatal deaths. The model explained $58 \%$ of neonatal deaths, with an accuracy of $93 \%$.

Conclusions: The risk factors associated to neonatal deaths show the importance of low birth weight prevention and a good prenatal and delivery care, in order to improve neonatal vitality at birth. The model had a good accuracy and a high power to explain the neonatal death.

Key-words: neonatal mortality; birth defects; infant, low birth weight; risk factors.
'Acadêmica do Departamento de Medicina da Universidade de Taubaté, Taubaté, SP, Brasil

${ }^{2}$ Professor assistente do Departamento de Medicina da Unitau e doutor em Saúde Pública pela Faculdade de Saúde Pública da Universidade de São Paulo, Taubaté, SP, Brasil
Endereço para correspondência:

Luiz Fernando C. Nascimento

Rua Durval Rocha, 500 - Vila Paraíba

CEP 12515-710 - Guaratinguetá/SP

E-mail: Ifcn@unitau.br

Recebido em: 14/6/2007

Aprovado em: 27/8/2007 


\section{Introdução}

A taxa de mortalidade neonatal é um indicador de saúde que, quando elevada, pode representar más condições de vida e dificuldade para acesso aos serviços de saúde, tanto no período pré-natal como no atendimento hospitalar ${ }^{(1)}$.

Os determinantes da mortalidade neonatal são complexos e variados, envolvendo fatores biológicos maternos e do recémnascido, assistenciais, que dizem respeito ao atendimento e à qualidade dos serviços de saúde, e socioeconômicos, pois podem se relacionar a dificuldades de acesso ao serviço de saúde, retardar o início do pré-natal e, de acordo com o nível de escolaridade, influenciar o resultado da gestação(2).

Dentre as causas da mortalidade neonatal, destacam-se o baixo peso ao nascer, a prematuridade, a presença de defeitos congênitos complexos e a asfixia neonatal ${ }^{(3,4)}$, sendo que tais fatores vêm sendo identificados como risco para o óbito neonatal em várias localidades do Brasil ${ }^{(4-7)}$. Por outro lado, as taxas de mortalidade neonatal também apresentam correlação espacial positiva com aglomerados intra-urbanos ou regionais ${ }^{(8,9)}$.

As taxas de mortalidade neonatal em 2003 foram 12,26 óbitos por 1.000 nascidos vivos no Brasil, 11,14 por mil na região Sudeste e 10,14 por mil no Estado de São Paulo ${ }^{(10,11)}$.

Visto que, na maioria das circunstâncias, o óbito neonatal é considerado um evento evitável pela utilização de tecnologias que estão disponíveis atualmente, a redução destes índices torna-se um desafio. Neste contexto, os objetivos deste trabalho foram identificar os fatores de risco para o óbito neonatal e estimar o seu papel por meio da técnica de regressão logística, para o ano de 2003, em Taubaté, SP.

\section{Métodos}

Trata-se de um estudo caso-controle, desenvolvido para identificar fatores associados ao óbito neonatal. O estudo foi realizado no município de Taubaté, São Paulo, o qual conta com uma população de cerca de 250.000 habitantes e se localiza às margens da Via Dutra, no Vale do Paraíba Paulista.

O estudo é baseado em dados vinculados de Declaração de Óbito (DO) e de Nascido Vivo (DNV). Foram considerados como casos todos os nascidos vivos que morreram antes de completar 28 dias de vida, no período de $1^{\circ}$ de janeiro de 2003 a 27 de janeiro de 2004, e que eram filhos de mães residentes neste município. Os controles foram os nascidos nos mesmos dias daqueles que morreram. Esta composição foi feita por meio do procedimento conhecido por linkage. No banco construído, foram agrupadas as informações de casos e de controles.
Os bancos de dados de nascidos vivos e de óbitos foram obtidos do portal da Secretaria Estadual da Saúde de São Paulo ${ }^{(10,11)}$. Os casos foram identificados por meio do número da DNV constante na DO. Desta forma, o banco construído para o estudo continha as informações sociodemográficas e do pré-natal da mãe (idade, estado civil, cor da pele, escolaridade, número de consultas, número de filhos vivos e número de filhos mortos) e informações do recém-nascido (peso ao nascer, sexo, duração da gestação, Apgar de um e cinco minutos e presença de anomalia congênita). Foi introduzida a variável "caso" para os óbitos e "controle" para os recémnascidos que estavam vivos até 28 dias de vida.

$\mathrm{Na}$ estratégia metodológica, como se esperava um pequeno número de óbitos neonatais, todos os controles foram selecionados para cada caso entre as crianças nascidas na mesma data do caso e que sobreviveram ao $28^{\circ}$ dia de vida. Os óbitos considerados no estudo seguem os seguintes critérios: nascimento ocorrido em 2003, local de nascimento e endereço de residência em Taubaté. Foram excluídos os recém-nascidos que morreram em 2003, mas nasceram em 2002.

A técnica de linkage foi utilizada equiparando cada DO com sua respectiva DNV, cruzando as informações obtidas em ambos os dados como a data de nascimento, peso, sexo e endereço de residência.

As variáveis sociodemográficas e assistenciais analisadas nesse estudo foram: idade materna, categorizada em adolescentes (até 20 anos de idade) e adultas; escolaridade materna, categorizada em até primeiro grau e outra classe para as mães com segundo e terceiro graus; paridade, categorizada em primeiro ou segundo filho e terceiro ou mais filhos; tipo de parto (cesáreo ou vaginal); número de consultas no pré-natal, classificada em até seis consultas e sete ou mais; e relato de óbito fetal.

As variáveis biológicas do recém-nascido analisadas foram: sexo; peso ao nascer, categorizado em baixo peso (menor que $2.500 \mathrm{~g}$ ) e normal; idade gestacional, classificada em pré-termo (até 37 semanas) e termo (37 a 41 semanas); Apgar de cinco minutos, classificado em menor que oito (representando uma menor vitalidade do recém-nascido) e igual ou maior que oito; e presença de defeito congênito.

A técnica utilizada foi a regressão logística - forward stepwise - para estimar o modelo preditivo para o óbito neonatal pelo programa SPSS 10.0. Foram inicialmente incluídas as variáveis $\operatorname{com} p<0,20$ para o nível que continha as variáveis socioeconômicas, demográficas e assistenciais, permanecendo aquelas que mantiveram $p<0,05$. Em segui$\mathrm{da}$, introduziram-se as variáveis do recém-nascido da menor Odds Ratio (OR) para a maior, permanecendo no modelo 
final as com $p<0,05$. A acurácia do modelo foi estimada pela curva ROC (Receive Operator Caracteristic Curve), com intervalo de confiança de $95 \%$ e obtido o valor do pseudo$\mathrm{R}^{2}$ de Nagelkerke.

Foram criadas tabelas com as variáveis independentes e a situação caso ou controle com os níveis de significância calculados pela técnica do qui-quadrado. Estes foram obtidos pelo cruzamento dos bancos de dados do Epi-Info versão 6.04 .

\section{Resultados}

Foram incluídos no estudo 392 recém-nascidos, sendo 34 óbitos neonatais ( $9 \%$ dos nascidos da amostra). Havia 198 recém-nascidos do sexo masculino ( $50 \%$ ), 60 com baixo peso (15\%) e 51 prematuros (13\%). A idade materna média foi 26 anos (desvio padrão de 6), variando entre 14 e 43 anos.

Os valores obtidos na análise univariada das OR brutas, dos intervalos de confiança e dos respectivos valores de

Tabela 1 - Fatores de risco para óbito neonatal com as respectivas OR brutas, os intervalos de confiança e o valor de $p$, para a população estudada em Taubaté, em 2003

\begin{tabular}{|c|c|c|c|c|c|}
\hline Variável & Casos & Controles & OR bruta & IC95\% & valor de $p$ \\
\hline \multicolumn{6}{|l|}{ Idade materna } \\
\hline Até 20 anos & 5 & 58 & 0,89 & $0,32-2,31$ & 0,77 \\
\hline Maior de 20 anos & 29 & 300 & & & \\
\hline \multicolumn{6}{|l|}{ Escolaridade materna } \\
\hline Até primeiro grau completo & 14 & 151 & 0,89 & $0,43-1,85$ & 0,83 \\
\hline Segundo grau e superior & 20 & 209 & & & \\
\hline \multicolumn{6}{|l|}{ Número de filhos } \\
\hline Até dois & 14 & 97 & 1,93 & $0,88-3,69$ & 0,10 \\
\hline Três ou mais & 20 & 268 & & & \\
\hline \multicolumn{6}{|l|}{ Tipo de parto } \\
\hline Vaginal & 19 & 186 & 1,18 & $0,55-2,54$ & 0,64 \\
\hline Cesáreo & 15 & 174 & & & \\
\hline \multicolumn{6}{|l|}{ Consultas pré-natal } \\
\hline Até seis & 17 & 56 & 5,38 & $2,82-12,12$ & $<0,001$ \\
\hline Sete ou mais & 17 & 301 & & & \\
\hline \multicolumn{6}{|l|}{ Relato de óbito fetal } \\
\hline Presença & 9 & 37 & 3,12 & $1,25-7,68$ & 0,005 \\
\hline Ausência & 25 & 321 & & & \\
\hline \multicolumn{6}{|l|}{ Sexo } \\
\hline Masculino & 16 & 184 & 0,84 & $0,39-1,79$ & 0,63 \\
\hline Feminino & 18 & 174 & & & \\
\hline \multicolumn{6}{|l|}{ Peso ao nascer } \\
\hline Baixo peso & 27 & 33 & 37,85 & $15,36-93,80$ & $<0,001$ \\
\hline Peso normal & 7 & 325 & & & \\
\hline \multicolumn{6}{|l|}{ Idade gestacional } \\
\hline Pré-termo & 25 & 26 & 35,46 & $15,00-83,81$ & $<0,001$ \\
\hline Termo & 9 & 332 & & & \\
\hline \multicolumn{6}{|l|}{ Apgar de cinco minutos } \\
\hline Menor que oito & 16 & 3 & 117,68 & $31,09-445,34$ & $<0,001$ \\
\hline Maior ou igual a oito & 16 & 353 & & & \\
\hline \multicolumn{6}{|l|}{ Presença de anomalia } \\
\hline Sim & 11 & 5 & 36,98 & $11,77-116,23$ & $<0,001$ \\
\hline Não & 21 & 353 & & & \\
\hline
\end{tabular}


p estão na Tabela 1. Pode ser visto que algumas variáveis, como idade materna, escolaridade e tipo de parto, não apresentaram significância estatística de acordo com o proposto na metodologia. Por outro lado, todas as variáveis do recémnascido apresentaram significância estatística.

A Tabela 2 mostra os valores das OR ajustadas pelas próprias variáveis do conjunto sociodemográfico e assistencial. Neste momento, a variável "número de filhos vivos” perdeu a significância estatística, a qual foi mantida pelas variáveis "número de consultas" e "relato de óbito fetal".

A Tabela 3 mostra os valores das OR ajustadas pelas próprias variáveis do conjunto de variáveis biológicas do recémnascido e as do conjunto sócio-demográfico. Neste caso, a prematuridade e as variáveis do conjunto sociodemográfico perderam a significância estatística.

A Tabela 4 mostra as OR e a constante do modelo final. O pseudo $\mathrm{R}^{2}$ resultou 0,582 , significando que estas variáveis explicam $58 \%$ dos óbitos neonatais deste estudo. O modelo também apresentou um teste de Hosmer Lemeshow significativo $\left(\chi^{2}=0,54\right.$ e $\left.p=0,46\right)$.

A curva ROC mostrou uma acurácia de 93\% [IC95\%=86-99], como é mostrado na Figura 1.

\section{Discussão}

Estudos de mortalidade neonatal podem auxiliar na avaliação da qualidade de vida de uma população, refletindo, em parte, a qualidade e o acesso aos serviços de saúde e também da assistência hospitalar à gestante e ao recém-nascido, sendo este estudo o primeiro sobre mortalidade neonatal em uma cidade do Vale do Paraíba Paulista.

Neste trabalho, a análise univariada não mostrou associação de algumas variáveis sociodemográficas e assistenciais e também de outras variáveis biológicas do recém-nascido

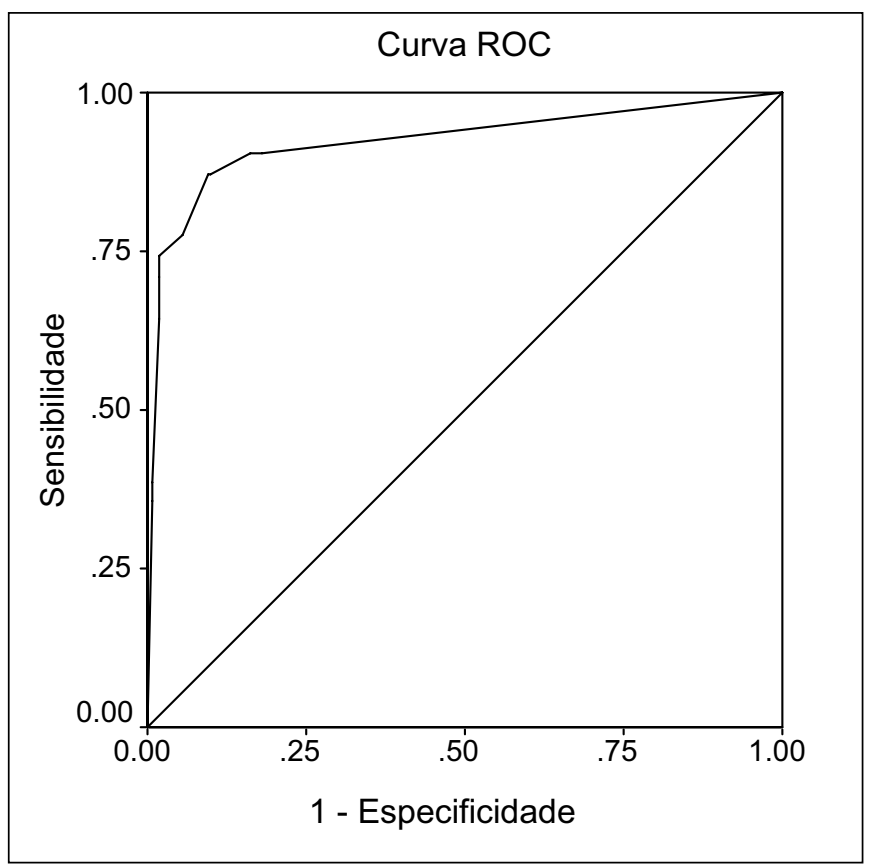

Figura 1 - Curva ROC para estimar a acurácia do modelo de regressão logística para óbito neonatal, com três variáveis independentes, em Taubaté, em 2003

Tabela 2 - Variáveis sociodemográficas e assistenciais com as OR ajustadas e respectivos intervalos de confiança de 95\% e valor de $p$

\begin{tabular}{lccc}
\hline & OR ajustadas & IC95\% & $\boldsymbol{p}$ \\
\hline Até seis consultas no pré-natal & 5,81 & $2,78-12,15$ & $<0,001$ \\
Relato de óbito fetal & 2,71 & $1,05-6,94$ & 0,038 \\
Até dois filhos & 1,36 & $0,63-2,94$ & 0,44 \\
\hline
\end{tabular}

Tabela 3 - Variáveis do recém nascido com as OR ajustadas, respectivos intervalos de confiança de 95\% e valor de $p$

\begin{tabular}{lccc}
\hline & OR ajustadas & IC95\% & $\boldsymbol{p}$ \\
\hline Baixo peso ao nascer & 13,79 & $3,49-54,58$ & $<0,001$ \\
Escore de Apgar $<8$ com cinco minutos & 13,34 & $2,57-69,26$ & 0,002 \\
Presença de defeito congênito & 12,29 & $1,96-76,99$ & 0,007 \\
Prematuridade & 2,34 & $0,62-8,88$ & 0,21 \\
\hline
\end{tabular}

Tabela 4 - Modelo final, com os coeficientes, as OR ajustadas, respectivos intervalos de confiança de $95 \%$ e o valor de $p$

\begin{tabular}{lcccc}
\hline & Beta & OR ajustadas & IC95\% & $\boldsymbol{p}$ \\
\hline Baixo peso ao nascer & 3,13 & 22,80 & $7,52-69,12$ & $<0,001$ \\
Escore de Apgar <8 com cinco minutos & 2,92 & 18,61 & $3,85-90,02$ & $<0,001$ \\
Presença de defeito congênito & 2,56 & 12,91 & $2,13-78,38$ & 0,007 \\
\hline
\end{tabular}

Constante $\left(\beta_{0}\right)=-4,35$; teste de Hosmer e Lemeshow: $\chi^{2}=0,54(p=0,46)$ 
com o óbito neonatal. No primeiro grupo de variáveis, a idade materna, categorizada em "adolescente" e "adulta", não mostrou significância estatística. O óbito neonatal se associou à idade mais jovem em estudo realizado no Rio de Janeiro, provavelmente por falta de atenção ao pré-natal, baixa escolaridade e pobreza ${ }^{(12)}$, e o mesmo ocorreu em Caxias do Sul, Rio Grande do Sul ${ }^{(6)}$. No entanto, os achados deste estudo coincidiram com os de Blumenau, Santa Catarina ${ }^{(4)}$, que também não mostrou a idade materna como fator de risco para o óbito neonatal.

A escolaridade materna não foi fator de risco, neste estudo, para o óbito neonatal, coincidindo com os resultados encontrados em Goiânia, Goiás ${ }^{(8)}$. Os valores encontrados contrariam os achados por Araújo et a $l^{(6)} \mathrm{e}$ Helena et $a l^{(4)}$, que demonstram ser a escolaridade materna relacionada a uma melhor situação socioeconômica, constituindo-se em um marcador obstétrico ${ }^{(13)}$. Talvez os achados destes estudos fossem diferentes, caso se considerasse as classes encontradas na DNV, pois, num estudo em Montes Claros, Minas Gerais, a mortalidade diminuiu com o aumento da escolaridade ${ }^{(5)}$.

O parto cesáreo, que poderia se mostrar como fator protetor, possivelmente pelo fato de estar associado a maiores níveis de escolaridade ${ }^{(13)}$ e a um melhor acesso aos serviços de saúde, tanto no âmbito ambulatorial como hospitalar, não mostrou significância estatística. Tal variável tem um papel discutível, sendo que nossos achados coincidem com alguns estudos ${ }^{(5,14)}$. Não foi estudado o tipo de hospital (se público ou privado) em que ocorreu o óbito, mas, talvez, no hospital privado o papel protetor do parto cesáreo pode ter uma explicação semelhante à acima referida.

As demais variáveis do grupo sociodemográfico e assistencial apresentaram significância estatística. O número maior de consultas no pré-natal foi fator protetor para o óbito neonatal, indicando a importância dos cuidados neste período no resultado da gravidez: um acompanhamento gestacional mais freqüente e rigoroso pode identificar precocemente e prevenir situações danosas para o recém-nascido. Os achados de nosso estudo são idênticos aos encontrados na literatura ${ }^{(4-6)}$.

Quanto ao número de filhos anteriores, o óbito esteve associado ao primeiro ou segundo filho, com chance duas vezes maior neste grupo. Este achado difere do encontrado em Caxias do Sul ${ }^{(6)}$, possivelmente por serem diferentes amostras, tanto no tamanho como no local onde foi realizado o estudo. Quanto ao relato de óbitos fetais anteriores, houve chance três vezes maior de óbito quando havia relato positivo, confirmando outros estudos ${ }^{(6-8)}$.
Das variáveis biológicas do recém-nascido, o sexo masculino é tido como fator de risco. A explicação de menor mortalidade no sexo feminino seria o amadurecimento mais precoce do pulmão fetal no sexo feminino, com diminuição de problemas respiratórios, que estão entre as principais causas de óbito neonatal ${ }^{(6,7)}$. Em nosso estudo, o sexo feminino aumentou em quase $20 \%$ a chance de óbito, mas este dado não foi estatisticamente significativo.

Prematuridade e baixo peso ao nascer são apresentados na literatura como importantes fatores de risco para o óbito neonatal ${ }^{(4-8,8,15)}$. No entanto, segundo Morais $\mathrm{Neto}^{(8)}$, estas duas variáveis não devem ser estudadas isoladamente, mas como mediadoras, por meio das quais agem outros determinantes, como nível socioeconômico materno, acesso aos serviços de saúde e qualidade dos mesmos, entre outros. Neste estudo de Taubaté, ambas as variáveis apresentaram significância estatística na análise univariada, sendo que a chance de óbito no neonato prematuro ou de baixo peso foi mais de 30 vezes maior. No entanto, quando realizada a análise multivariada, a prematuridade perdeu a significância estatística, sendo ajustada pelas outras variáveis biológicas do recém-nascido (possivelmente pela inter-relação da prematuridade com o baixo peso ao nascer e com os baixos escores de Apgar). Outros estudos contradizem nossos achados ${ }^{(5,8,15,16)}$. Permaneceu com significância o baixo peso ao nascer, visto que tais recémnascidos são mais vulneráveis a problemas como imaturidade pulmonar e transtornos metabólicos, coincidindo com os resultados de outros trabalhos ${ }^{(3-8,15)}$.

Outros fatores biológicos que aumentam a chance de óbito neonatal foram: escore de Apgar de cinco minutos menor que oito, representando menor vitalidade do recém-nascido, e a presença de defeito congênito, não sendo separados em defeitos maiores e menores. Quanto à menor vitalidade do recém-nascido, o valor de corte para o escore de Apgar escolhido neste estudo indica que, quando acima dele, o neonato nasceu em boas condições, sem hipóxia ${ }^{(17)}$. A associação entre valores de Apgar abaixo de oito e mortalidade neonatal é coincidente com outros estudos ${ }^{(4-7,15)}$ e pode estar relacionada à qualidade do atendimento do neonato na sala de parto. Após ajustes por todas as variáveis do modelo, este fator se mostrou muito importante, pois a presença de Apgar menor que oito aumentou a chance de óbito em quase 20 vezes. A presença de defeito congênito, por sua vez, foi fator de risco para o óbito neonatal e, mesmo após ajuste, a chance de óbito neonatal foi 13 vezes maior nos portadores de anomalias congênitas, coincidindo com os achados em Blumenau ${ }^{(4)}$. 
As possíveis limitações deste estudo se referem à análise de dados de somente um ano (2003) e ao fato de não haver identificação do tipo de hospital (público ou privado). Apesar disso, é possível concluir que medidas para diminuir a incidência de baixo peso ao nascer, propiciar melhor atendimento na sala de parto para diminuir a ocorrência de recém-nascidos com escore de Apgar inferior a oito, além da identificação e da abordagem precoces de defeitos congênitos poderão diminuir a chance de óbito neonatal.
Assim, foi possível identificar e quantificar fatores de risco para óbito neonatal em Taubaté, no ano de 2003: baixo peso, menor vitalidade do recém-nascido e presença de anomalia congênita. $\mathrm{O}$ modelo mostra que um neonato portador de todas estas características teria quase $98 \%$ de probabilidade de morrer antes de completar 28 dias e outro, que não apresentasse nenhuma delas, teria a probabilidade de cerca de $2 \%$ de óbito.

\section{Referências bibliográficas}

1. Victora CG, Barros FC. Infant mortality due to perinatal causes in Brazil: trends, regional patterns and possible intervention. São Paulo Med J 2001;119:33-42.

2. Machado CJ, Hill K. Determinants of neonatal and post-neonatal mortality in the City of São Paulo. Rev Bras Epidemiol 2003;6:345-58.

3. Almeida MF, Novaes HMD, Alencar GP, Rodrigues LC. Mortalidade neonatal no Município de São Paulo: influência do baixo peso ao nascer e de fatores sócio-demográficos e assistenciais. Rev Bras Epidemiol 2002;5:93-107.

4. Helena ETS, Sousa CA, Silva CA. Risk factors for neonatal mortality in Blumenau, Santa Catarina: linkage between database. Rev Bras Saude Mater Infant. 2005;5:209-17.

5. Martins EF, Velasquez-Meléndez G. Determinantes da mortalidade neonatal a partir de uma coorte de nascidos vivos, Montes Claros, Minas Gerais, 19971999. Rev Bras Saúde Mater Infant 2004;4:405-12.

6. Araújo BF, Bozzetti MC, Tanaka AC. Early neonatal mortality in Caxias do Sul: a cohort study. J Pediatr (Rio J) 2000;76:200-6.

7. Sarinho SW, Filho DA, Silva GA, Lima MC. Risk factors for neonatal death in Recife: a case-control study. J Pediatr (Rio J) 2001;77:294-8.

8. Morais Neto OL, Barros MBA, Martelli CMT, Silva SA, Cavenaghi SM, Siqueira Jr JB. Diferenças no padrão de ocorrência de mortalidade neonatal e pós-neonatal no município de Goiânia, Brasil, 1992-1996: análise espacial para identificação de áreas de risco. Cad Saúde Pública 2001;17:1241-50.
9. Nascimento LFC, Batista GT, Dias NW, Catelani CS, Becker D, Rodrigues L. Análise espacial da mortalidade neonatal no Vale do Paraíba, 1999-2001. Rev Saúde Pública 2007;41:94-100.

10. Brasil - Ministério da Saúde - DATASUS [homepage on the Internet]. Informações de Saúde - Estatísticas Vitais - Mortalidade e Nascidos Vivos: nascidos vivos desde 1994 [cited 2007 Apr 2]. Available from: http://tabnet. datasus.gov.br/cgi/tabcgi.exe?sim/cnv/infuf.def

11. Brasil - Ministério da Saúde - DATASUS [homepage on the Internet]. Informações de Saúde- Estatísticas Vitais- Mortalidade e Nascidos Vivos: óbitos infantis desde 1979 [cited 2007 Apr 2]. Available from: http://tabnet.datasus. gov.br/cgi/tabcgi.exe?sinasc/cnv/infuf.def

12. Andrade CLT, Szwarcwald CL. Análise espacial da mortalidade neonatal precoce no Município do Rio de Janeiro, 1995-1996. Cad Saúde Pública 2001;17: 1199-210.

13. Haidar FH, Oliveira UF, Nascimento LFC. Escolaridade materna: correlação com os indicadores obstétricos. Cad Saúde Pública 2001;17:1025-9.

14. Duarte G, Coltro PS, Bedone RV, Nogueira AA, Gelonezzi GM, Franco LJ et al. Trends in the modes of delivery and their impact on perinatal mortality rates. Rev Saúde Pública 2004;38:379-84.

15. Almeida MF. Mortalidade neonatal em Santo André [tese de doutorado]. São Paulo (SP): Faculdade de Saúde Pública da USP; 1994.

16. Berkowitz GS, Papiernik E. Epidemiology of preterm birth. Epidemiol Rev 1993;15: 414-43.

17. Segre CMA. Atendimento ao recém-nascido em sala de parto. In: Segre CMA, Armellini PA. Recém-nascido. São Paulo: Sarvier, 1981. 